\title{
MigRATIONS ET MARCHÉ DU TRAVAIL DANS L'ESPACE EUROPÉEN
}

\author{
El Mouhoub Mouhoud \& Joël Oudinet ${ }^{1}$
}

Date de réception de l'article: 27 novembre 2004

Date d'acceptation pour publication: 20 avril 2006

RÉSUMÉ. Cet article analyse le rôle et les déterminants des migrations de main-d'œuvre sur les marchés du travail des pays de l'Union européenne selon le degré de qualification des migrants, leurs caractéristiques sur les marchés du travail et leur origine intra- ou extra-communautaire. L'immigration dans les pays de l'Union apparaît moins sensible aux variables de déséquilibre du marché du travail, telles que le niveau de salaire relatif et le taux de chômage relatif qu'aux variables structurelles telles que les effets de réseaux ou les différences d'aménités. Toutefois, les migrants qui viennent des pays non membres de l'Union européenne sont davantage influencés par les différences de salaires entre les pays, dans leur choix du pays d'accueil, que les migrants d'origine intra-communautaire.

Classification JEL : J6; J61.

Mots-clefs: Migration; flux migratoires; mobilité; Union européenne; réseaux.

ABStRACt. The aim of this article is to analyse the role and determinants of labour migration in EU labour markets, depending on skill levels, the characteristics of migrants in the labour market and their origin inside or outside the Union. Immigration to EU countries is less sensitive to variables representing labour market imbalances, such as relative wage levels and relative unemployment rates, than to variables representing the network effects which reduce migration costs. However, the effect of these variables on immigration flows varies strongly from country to country and remains limited overall.

JEL Classification: J6; J61. Keywords: Migration; Flow of Immigrants; Mobility; European Union; Networks.

1. Auteur correspondant: El Mouhoub Mounoud, Professeur à I'Université Paris XIII et directeur du Centre d'Économie de Paris-Nord (CEPN-CNRS) (mouhoud@seg.univ-paris13.fr).

Joël OUdINET, Maître de Conférences à I'Université Paris XIII et membre du Centre d'Économie de Paris-Nord (CEPN-CNRS). 
Cet article s'intéresse aux migrations intra-européennes à la suite des interrogations sur le rôle de la mobilité du travail en tant qu'instrument d'ajustement des économies intégrées dans une union monétaire telle que la zone euro. Les analyses théoriques en termes de zones monétaires optimales ont longtemps constitué le cadre de référence théorique de l'impact des migrations sur le fonctionnement des zones monétaires intégrées (Mundell, 1957, Blanchard et Muet, 1993). Lorsque les économies ne sont pas complètement intégrées, le risque qu'elles soient affectées négativement par un choc spécifique est élevé, en particulier pour celles qui sont périphériques au sein de l'union monétaire (Bayoumi et Prasad, 1995). En l'absence d'autres instruments (change, budget, mobilité du capital...), la flexibilité du marché du travail est théoriquement supposée jouer ce rôle d'instrument d'ajustement des économies. L'économie affectée négativement par un tel choc asymétrique voit sa production et son emploi décliner, mais elle peut relancer sa croissance grâce aux gains de parts de marché obtenus par un mécanisme de désinflation compétitive. Pour autant, les travaux empiriques montrent que, dans la plupart des cas, les flexibilités des prix et des salaires n'ont pas d'impact significatif (Cadiou, Guichard et Maurel, 1999; Mazier, Oudinet et Saglio, 2002). En tant qu'instrument d'ajustement efficace face à ces éventuels chocs asymétriques, il ne reste alors que le rôle des migrations de travailleurs, peu analysé pour l'Union européenne, au contraire des États-Unis (Blanchard et Katz, 1992).

Le pays, par exemple touché par un choc de demande, réduit le nombre de ses chômeurs en les laissant partir vers les pays qui ne sont pas affectés et où les salaires et les perspectives d'emploi sont plus élevés. II y a donc une diffusion géographique au sein de l'union monétaire de ce choc négatif. Mais pour que les migrations jouent ce rôle d'ajustement, deux conditions, au moins, doivent être réunies. D'abord, le volume des flux de migration doit être relativement important pour que l'impact ne soit pas négligeable sur les marchés du travail des pays membres de l'union monétaire. Ensuite, les élasticités des flux migratoires aux variables de déséquilibre du marché du travail (différences de taux de chômage, dynamique de l'emploi, différences de salaires) doivent être suffisamment élevées. En d'autres termes, les chômeurs sont très sensibles aux différences de salaires ou de perspectives d'emplois entre les pays de l'union monétaire pour leur choix d'émigration (Harris et Todaro, 1970).

Dans les faits, d'autres facteurs déterminent les comportements des migrants qui sont davantage liés aux effets de réseaux entre les familles ou tribus de migrants (Sjastad, 1962) et à des facteurs spécifiques (différences d'aménités, de situations sociales et politiques, Graves, 1980) ne concernant pas nécessairement les marchés du travail. Par exemple, un citoyen d'un pays européen, au chômage à la suite d'un choc négatif, ne recherchera pas nécessairement à émigrer pour retrouver un emploi dans un pays dans lequel peu de ses concitoyens se seraient installés précédemment (absence de réseaux) ou encore dans lequel le système de protection sociale serait moins favorable en dépit de meilleures conditions sur le marché du travail. 
Ces deux conditions de volume et de sensibilité seront analysées dans le cadre de notre problématique d'ajustement des marchés du travail grâce à deux bases de données différentes utilisées de manière complémentaire. Les travaux empiriques permettant de comparer les caractéristiques et les comportements des migrants sur les marchés du travail des pays de I'Union européenne sont, en effet, peu fréquents compte tenu de la difficulté d'avoir des données homogènes.

L'objectif de cet article est donc de répondre à une question rarement traitée d'un point de vue comparatif : quel est le poids des variables du marché du travail dans le choix de la localisation du pays de destination de la migration, en comparaison avec toutes les autres variables (comme les aménités des pays ou les réseaux constitués) qui peuvent intervenir?

Traiter cette question des migrations s'avère en fait complexe car il existe une multiplicité de logiques et de caractéristiques des migrations selon le pays d'accueil (politiques d'immigration diverses ${ }^{2}$, besoins économiques différents) et selon l'origine intra ou extra-communautaire du migrant. Est-ce qu'il y a une différence entre les migrants de nationalité européenne et les non-Européens, entre ceux qui partent pour la première fois et ceux qui sont en situation de mobilité répétée? Est-ce que le comportement diffère si l'on émigre directement vers le pays ciblé ou bien si l'on passe d'abord par un pays considéré comme un pays relais?

Compte tenu de cette complexité, il est nécessaire, avant d'effectuer une estimation du rôle respectif des deux catégories de déterminants, de préciser l'ampleur, les pays d'origine et les pays de destination des migrants au sein de l'Union européenne (UE). Le rôle des variables du marché du travail selon différentes catégories de migrants et différents types de pays d'accueil est examiné ensuite.

\section{LES DÉTERMINANTS DES MIGRATIONS}

\section{SUR LES MARCHÉS DU TRAVAIL DE L'UNION EUROPÉENNE}

L'une des valeurs ajoutées de cet article provient de l'utilisation de nouvelles statistiques issues de l'enquête Labor Force Survey (LFS), organisée par Eurostat (depuis 1992 pour certains pays européens et 1995 pour les autres). Dans cette enquête, les réponses aux questions relatives au pays de résidence l'année précédente permettent d'obtenir des données homogènes de migration pour les pays membres de l'UE. Les bases de données traditionnelles de flux migratoires sont, au contraire, hétérogènes puisque les critères d'enregistrement et les définitions des migrants sont différents selon les pays ${ }^{3}$.

Par contre, la disponibilité des données de l'enquête LFS sur une période insuffisamment longue, ainsi que l'absence de données concernant les flux d'émigration n'autorisent pas I'utilisation de la base LFS pour les estimations des déterminants. Seules des données d'immi-

2. Voir sur ce sujet Mat et Rodier (2003) entre autres.

3. Voir l'ANNEXE 1 pour de plus amples précisions sur les bases de données et Eurostat $(1998,2002)$. 
gration sont disponibles ${ }^{4}$. C'est pourquoi les données de NewCronos d'Eurostat sont utilisées à partir des paragraphes 2 et 3 . Elles sont en outre compatibles avec celles de LFS (voir Rendall et alii, 2003 pour le cas du Royaume-Uni).

Avant d'estimer la sensibilité des variables déterminantes des migrations au niveau des pays européens, il convient d'abord de fournir des éléments d'appréciation sur l'ampleur et les caractéristiques des migrations intra-européennes. Nous mettons ensuite l'accent sur les liens entre les flux de migration d'une année sur l'autre afin de repérer les éventuels effets de réseaux qui structurent les migrations dans les différents pays européens. II est proposé enfin une analyse économétrique des déterminants des migrations liés aux marchés du travail (taux de chômage relatif et taux de salaire relatif) en utilisant une typologie des migrations qui permet de distinguer les immigrants des émigrants et les comportements spécifiques des nationaux.

\section{L'ampleur des migrations en Europe: relance et nouvelles mobilités}

\section{Faiblesse historique des flux intra-communautaires, nouvelles dynamiques extra-communautaires}

Si les grands pays d'accueil (Allemagne, Royaume-Uni, France) continuent de recevoir la majorité des immigrants de I'Union européenne, on assiste depuis le milieu des années quatre-vingt à une reprise des flux d'immigration qui s'accélère à partir de la fin des années quatre-vingt-dix. Cette croissance nouvelle est permise par de nouveaux pays d'accueil. ॥ s'agit des pays d'Europe du sud devenus récemment des pays d'immigration nette (Italie, Espagne, Portugal, Grèce) et d'autres pays périphériques comme I'Irlande et la Finlande (Bailly, Mouhoud et Oudinet, 2003 ; Dumont et Garson, 2004).

Malgré cela, les flux migratoires intra-communautaires restent faibles en comparaison avec ceux qui existent entre les quatre grandes régions américaines (TABLEAU 1). Hormis le cas particulier du Luxembourg, les taux d'immigration des Européens (nombre total d'immigrants de I'UE sur la population totale du pays d'accueil) ne dépassent pas 0,25\% de la population totale et sont souvent inférieurs à $0,1 \%$ alors que les taux de migration internes aux ÉtatsUnis sont de l'ordre de 0,8 à 1,6\%.

L'analyse des flux réalisée à partir du pays de résidence l'année précédente, et non pas à partir de la nationalité du migrant, montre que les migrations internes à l'Union européenne ne doivent pas être réduites aux seuls migrants communautaires. Des migrants de nationalité non-communautaire, ainsi que les nationaux contribuent par leurs mobilités aux migrations intra-communautaires et doivent, par conséquent, être comptabilisés pour caractériser cette

4. La reconstitution de données d'émigrants n'est pas réalisable dans le cadre de cette enquête LFS qui ne prend en compte que les arrivées dans les pays membres de l'UE. Les flux d'émigration reconstitués seraient alors sous-estimés. Par exemple, les Français qui émigrent vers des pays non membres de l'UE ne sont pas comptabilisés. En outre, même en se limitant aux seules migrations intra-communautaires, la reconstitution sous-estimerait aussi le nombre réel d'émigrants vers les pays de l'UE car seules les nationalités les plus nombreuses sont distinguées alors que toutes les autres sont comptabilisées dans la catégorie "autres Union européenne". 
Tableau 1 - Taux d'immigration et d'émigration

En \% de la population totale dans les régions américaines et dans les grands pays européens

\begin{tabular}{|c|c|c|c|c|c|c|}
\hline \multirow[b]{2}{*}{ États-Unis } & \multicolumn{3}{|c|}{ Immigrants intra/POP } & \multicolumn{3}{|c|}{ Émigrants intra/POP } \\
\hline & Moy & $\max$ & Min & Moy & $\max$ & Min \\
\hline Middlewest & 1,32 & 1,72 & 1,05 & 1,49 & 1,98 & 0,99 \\
\hline Northeast & 0,84 & 1,00 & 0,61 & 1,37 & 1,83 & 1,16 \\
\hline South & 1,58 & 1,89 & 1,28 & 1,23 & 1,60 & 1,01 \\
\hline West & 1,62 & 2,08 & 1,16 & 1,45 & 1,89 & 1,14 \\
\hline Union européenne & \multicolumn{3}{|c|}{ Immigrants européens/POP } & \multicolumn{3}{|c|}{ Émigrants européens/POP } \\
\hline Belgique & 0,25 & 0,28 & 0,18 & 0,16 & 0,23 & 0,12 \\
\hline Allemagne & 0,17 & 0,22 & 0,14 & 0,15 & 0,19 & 0,12 \\
\hline Danemark & 0,10 & 0,15 & 0,06 & 0,07 & 0,12 & 0,05 \\
\hline Espagne & 0,02 & 0,08 & 0,01 & & & \\
\hline Finlande & 0,02 & 0,03 & 0,01 & 0,01 & 0,02 & 0,01 \\
\hline France & 0,02 & 0,04 & 0,01 & & & \\
\hline Grèce & 0,05 & 0,10 & 0,03 & & & \\
\hline Irlande & 0,24 & 0,39 & 0,10 & & & \\
\hline Italie & 0,02 & 0,03 & 0,01 & & & \\
\hline Luxembourg & 1,76 & 2,00 & 1,31 & & & \\
\hline Pays-Bas & 0,11 & 0,14 & 0,10 & 0,06 & 0,07 & 0,05 \\
\hline Portugal & 0,02 & 0,05 & 0,00 & & & \\
\hline Suède & 0,06 & 0,10 & 0,04 & 0,05 & 0,08 & 0,03 \\
\hline Royaume-Uni & 0,07 & 0,18 & 0,04 & 0,04 & 0,08 & 0,02 \\
\hline
\end{tabular}

Note: Flux intra-régions américaines et flux des communautaires.

Sources: Pour les États-Unis, données 1981-97 provenant de I'US Bureau of the Census, et pour les pays européens, données 1985-99 provenant d'Eurostat (NewCronos).

migration. À partir de l'enquête LFS (voir les détails et le tABLEAU A1.2 en ANNEXe 1), on s'aperçoit que l'ampleur de la migration intra-communautaire est fortement sous-estimée si l'on se limite aux seuls migrants de nationalité d'un pays membre de I'Union européenne et que l'on néglige les retours de nationaux et les migrations répétées de non-communautaires (respectivement colonne 3 et 5, TABLEAU 2).

Alors que la part des Européens dans l'immigration en Europe est de seulement $19 \%$ (colonnes 1 et 2, TABLEAU 2), plus d'un tiers des migrations globales s'effectuent au sein de I'Union européenne (38\%), les deux autres tiers de migrants provenant des pays extérieurs aux Quinze de I'Union européenne (62\%). En outre, plus récemment, la migration intraeuropéenne connaît une nette augmentation (+ $14 \%$ ) bien que la migration d'origine extracommunautaire demeure plus dynamique (+ $21 \%$ ). L'immigration intra-communautaire correspond donc au double de l'immigration des Européens, calculée dans le tABLEAU 1. Mais pour autant, les taux internes à l'Union européenne, même corrigés, restent près de 8 à 10 fois inférieurs aux taux de migration entre les régions américaines. 


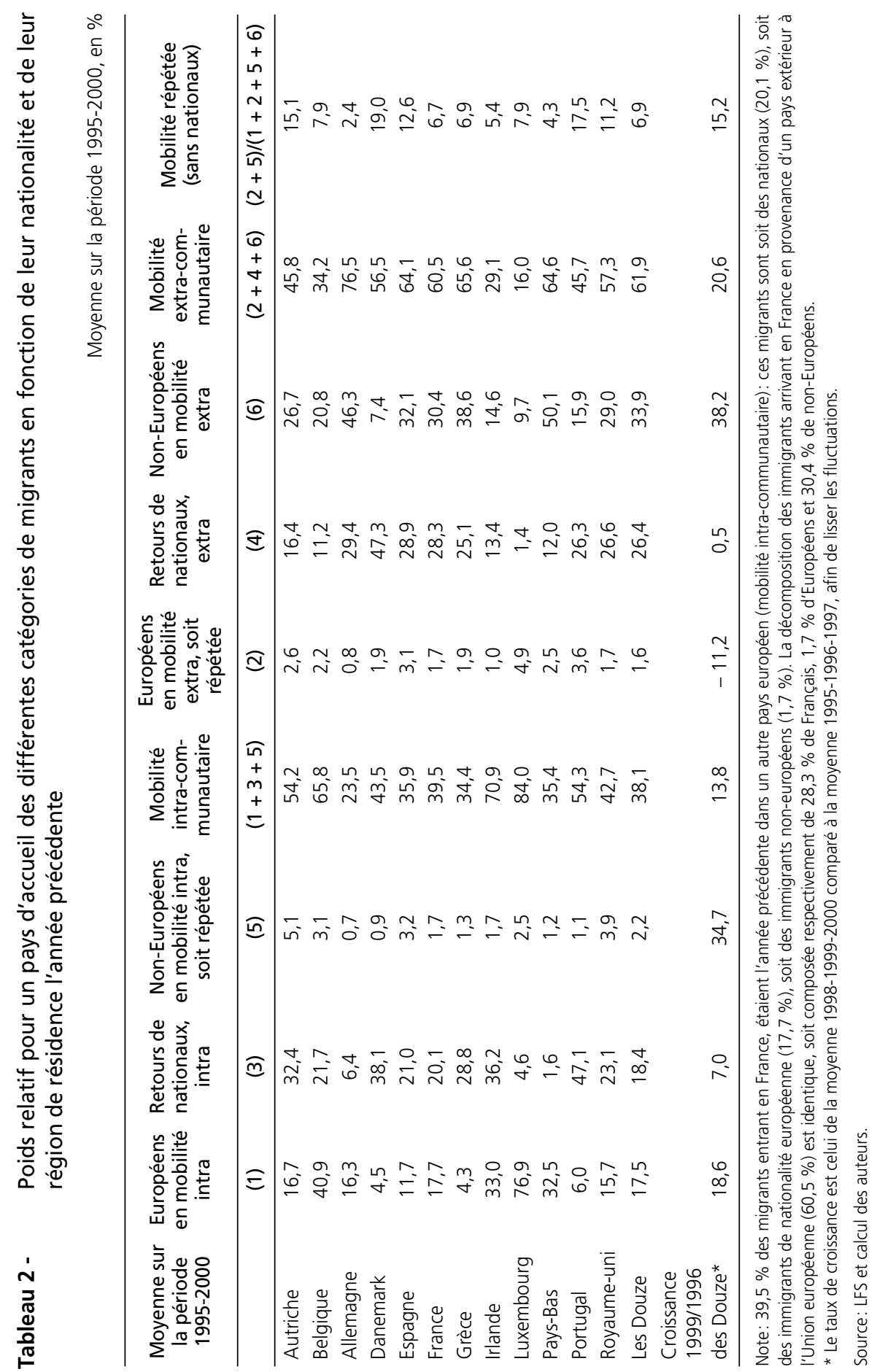




\section{Diversification des pays d'accueil et migrations répétées}

La situation des pays européens en tant que pays d'accueil est en fait très diversifiée. Certains accueillent majoritairement des migrants intra-communautaires, comme le Luxembourg (84\%), I'Irlande (71\%), la Belgique (64\%), l'Autriche et le Portugal (tableau 2). Les migrants arrivant en Allemagne viennent par contre majoritairement de l'extérieur de I'Europe (77\%), ainsi que ceux qui immigrent en Grèce (66\%), aux Pays-Bas (65\%), en Espagne $(65 \%)$ et en France (60\%).

Les caractéristiques des migrants ont également changé: on assiste à une montée des migrations qualifiées (Dumont et Le Maître, 2005) et au développement d'un phénomène nouveau, les migrations répétées, que l'exploitation de l'enquête LFS permet de mettre en évidence (Mouhoud et Oudinet, 2003).

La situation de migration répétée consiste pour un migrant à quitter son pays d'origine (I'Ukraine par exemple) et à résider dans un pays européen (I'Allemagne par exemple) après avoir séjourné dans un autre pays européen (par exemple le Portugal) $)^{5}$. La migration est dite répétée dans la mesure où le migrant opère au moins sa seconde émigration. Dans cet exemple, il s'agit de la migration répétée d'un migrant non-communautaire dans l'espace de I'Union européenne (colonne 5, TABLEAU 2). Un autre exemple de migration répétée serait celui d'un migrant communautaire (par exemple un Français) qui résiderait au moment de l'enquête en Espagne après avoir séjourné dans un autre pays non-européen (colonne 2, TABLEAU 2). L'enquête LFS, ne permet de construire et de mesurer que ces deux catégories de migration répétée et sous-estime donc l'ensemble du phénomène ${ }^{6}$. Ce type de migration, certes encore faible, reflète néanmoins un changement dans la dynamique et les caractéristiques des migrations et préfigure le développement des circulations migratoires.

Sur cette base, on observe que les migrations répétées, de plus en plus fréquentes, concernent plutôt les migrants en provenance des pays non membres de l'Union européenne. Pour l'ensemble des pays de l'Union européenne, la part des migrations répétées est de $7 \%$ (dernière colonne, TABLEAU 2). Le Portugal, l'Espagne, le Danemark et l'Autriche sont particulièrement choisis par les migrants qui sont en mobilité répétée, mais pour le Portugal et l'Espagne à un degré moindre, cela est essentiellement le fait des migrants européens.

L'analyse de ce phénomène révèle une nette différence des comportements des migrants selon leur nationalité. Les migrants non-communautaires tendent à utiliser les pays périphériques (Espagne, Portugal, Grèce...) pour atteindre les grands pays d'accueil du centre de I'Union européenne. Si l'ampleur de la migration répétée des non-européens est encore relativement faible, cette catégorie est en forte croissance sur les dernières années (+ $35 \%$

5. Dans cet exemple, l'Allemagne peut être qualifié de pays cible tandis que le Portugal est caractérisé de pays relais. 6. On ne peut pas, en revanche, isoler le migrant communautaire (par exemple un Français) qui résiderait au moment de l'enquête en Espagne après avoir séjourné aussi dans un autre pays de I'Union européenne (par exemple le Royaume-Uni). De même, un autre cas très spécifique de migration répétée ne peut être décelé, celui du retour de certains nationaux par exemple un Français revenant en France d'Allemagne après avoir séjourné précédemment dans un autre pays (par exemple le Royaume-Uni). 
entre 1996 et 1999) (TABLEAU 2). Un clivage semble se dessiner entre les pays qui jouent le rôle de pays cibles et ceux qui jouent le rôle de pays relais, clivage qui reflète des différences dans les besoins économiques et dans les politiques d'immigration des pays.

La situation des Européens en migration répétée est due à un autre aspect de la migration plus généralement liée à la mobilité des personnes diplômées (Bailly, Mouhoud et Oudinet, 2003). Les migrants communautaires se déplacent plutôt à l'intérieur des firmes multinationales et tendent à partir des pays du centre pour aller vers les pays périphériques de I'UE (Portugal, Grèce, Espagne et Danemark).

Au sein de l'espace européen, il existe donc une multiplicité de logiques et de caractéristiques des migrations. La relance de l'immigration intra-européenne (plus d'un tiers du total des migrations) est essentiellement stimulée par les non-Européens qui peuvent choisir de s'installer tout d'abord dans certains pays considérés comme des relais avant d'atteindre leur destination dans un autre pays européen. Les nouveaux pays d'accueil à la périphérie de I'Union européenne semblent jouer ce rôle. À l'inverse, les migrants européens font plutôt le choix des pays de la périphérie. Pour quelles raisons, ces différents types de migrants choisissent-ils tel ou tel pays européen?

\section{Les déterminants des migrations: des effets de réseaux dominants}

\section{Un modèle conciliant les facteurs liés aux marchés du travail et les facteurs structurels.}

Nous nous intéressons aux variables qui déterminent le choix du pays de destination des émigrants. II s'agit d'un arbitrage pour des personnes qui ont déjà fait le choix d'émigrer. Parmi les facteurs déterminants de ce choix, on peut distinguer ceux qui sont liés aux déséquilibres du marché du travail, et ceux qui dépendent de facteurs davantage structurels ou d'effets de réseaux des communautés de migrants dans divers pays d'accueil.

Un modèle structurel de déséquilibre des marchés du travail (équations 1 à 5) qui s'inspire d'Harris et Todaro (1970) est complété par les apports de Graves (1979) sur les différences d'aménités, afin de répondre à la question des rôles respectifs des variables du marché du travail et des autres variables structurelles (aménités, réseaux...) (Detang-Dessendre et Jayet, 2000). En outre, les coûts de migration, et tout particulièrement les coûts liés à l'incertitude de l'information relative aux pays d'accueil, justifient une inertie des décisions migratoires formalisée à partir d'un processus d'ajustement dichotomique (équation 6) (Greenwood, 1985; Stark et Bloom, 1985).

Les salaires et le taux de chômage comme variables de déséquilibre des marchés du travail

Dans notre modèle de base, les migrations sont une réaction aux déséquilibres sur et entre des marchés du travail, à l'instar des travaux initiés par Harris, Todaro (1970) et synthétisés par Greenwood (1975). Le migrant potentiel compare les espérances de rémunération des 
pays concurrents dont le pays d'origine $\left(w_{*}^{e}\right)$ et l'espérance de la rémunération dans la région de destination $\left(w_{i}^{e}\right)$. II choisit de migrer dans un pays i si l'espérance du salaire est supérieure à l'espérance des salaires des pays concurrents (dont le pays d'origine) et aux coûts relatifs (entre différentes localisations) de migration (c).

$$
w_{i}^{e}-w_{*}^{e}>c
$$

Dans le modèle statique d'Harris et Todaro (1970), le salaire anticipé est égal au produit du salaire par la probabilité instantanée de trouver un emploi:

$$
w_{i}^{e}=\left(1-u_{i}\right) w_{i}
$$

Le taux de chômage a un effet négatif sur l'immigration en réduisant la probabilité de trouver un emploi ( $1-u_{i}, u$ étant le taux de chômage) dans le pays d'accueil, et donc en diminuant l'espérance du salaire.

\section{Intégration des aménités dans ce modèle de déséquilibre}

D'autres facteurs sont mis en avant par Graves (1979) dans les modèles où les marchés sont proches de l'équilibre. Les changements des ressources et des préférences durant leur cycle de vie incitent les agents à migrer pour consommer d'autres aménités. Les couples avec enfants sont plus sensibles aux structures publiques (crèches, écoles...), alors qu'en vieillissant les structures de santé et le climat deviennent primordiaux (équations 3 et 4).

Mais le rôle majeur des aménités n'est pas forcément lié à l'hypothèse d'équilibre, comme celui du marché du travail serait associé à I'hypothèse du déséquilibre. Les aménités peuvent être le principal déterminant des migrations dans une économie en déséquilibre; de même les emplois peuvent être le principal déterminant dans une économie en équilibre (Evans, 1990).

Le modèle se généralise donc à des comparaisons d'utilité et à des coûts non monétaires:

$$
U^{e}\left(w_{i}, x_{i}\right)-U^{e}(W *, x *)>c
$$

Les variables $x_{*}$ et $x_{i}$ correspondent à des éléments non monétaires de l'utilité, correspondant à chaque localisation (aménités): jouissance de bien publics, climat. On voit notamment que I'accès aux biens publics, présent dans la fonction d'utilité des agents (variables $X_{*}$ et $x_{j}$ ), peut constituer un argument important de la migration, pour peu que ces biens publics entrent significativement dans le bien-être (Graves, 1979). Dans un cadre intertemporel, on écrira alors le comportement de migration de la façon suivante:

$$
\int_{t}^{T}\left[U^{e}\left(w_{i, h}\right)-U^{e}\left(w^{*}, h\right)\right] e^{-\rho(t-h)} d h>\int_{t}^{T} c_{h}^{e} e^{-\rho(t-h)} d h
$$

cela pour une migration définitive envisagée à la date $t$, à l'horizon $\mathrm{T}^{7}$.

7. Nous avons ici négligé les variables $x_{1}$ et $x_{2}$, en reportant leur effet dans un étalement dans le temps des coûts de migration. 
Un modèle intégrant des variables du marché du travail et des aménités

À la suite des équations 1 à 4, en restant dans un cadre de déséquilibre, on peut tenter de formuler un modèle qui intègre des variables relevant du marché du travail et d'autres des aménités, afin de répondre à la question des rôles respectifs de ces facteurs (Jayet, 1996; Detang-Dessendre et Jayet, 2000).

$$
t M_{i, t}{ }^{e}=\left(\frac{M_{i, t}}{P O P_{i, t}}\right)=\Phi\left[\frac{w_{i, t}}{W_{*, t}}, \frac{U_{i, t}}{U_{*}, t}, \eta_{i}\right]
$$

avec $i=1, \ldots k$ pays européens,

$I M_{i}=$ flux d'immigration dans un pays $i$,

$P O P_{i}=$ population totale d'un pays i,

$W_{i}=$ salaire du pays i d'immigration en PPA,

$W^{*}=$ salaire moyen pondéré de I'UE en PPA,

$U_{i}=$ taux de chômage du pays i d'immigration,

$U^{*}=$ taux de chômage moyen pondéré de l'UE,

$\eta_{i}=$ effet fixe du pays i.

Les deux variables marquant le déséquilibre sur les marchés du travail, le salaire et le taux de chômage, apparaissant dans l'équation (2) sont présentes. Comme les marchés du travail sont interdépendants, les variables apparaissent d'une manière relative. Le salaire du pays $d^{\prime}$ accueil $i$ est divisé par le salaire moyen des pays concurrents $W^{*}$. Ainsi, une augmentation du salaire dans les pays concurrents plus forte que celui du pays d'accueil i réduira l'attrait de ce dernier. L'effet est inverse pour le taux de chômage qui baisse l'espérance de revenu dans le pays d'accueil.

À l'instar des équations (3) et (4), les aménités et autres variables structurelles sont appréhendées à travers les effets fixes spécifiques au pays d'accueil. Ces variables ne se modifient que très peu (les différences de climat par exemple n'évoluent quasiment pas).

\section{Coûts de migration, coûts d'information et incertitude}

L'importance des coûts de migration, monétaires et psychiques, et l'inertie des décisions migratoires liée à l'incertitude de l'information sont des facteurs qui compromettent l'efficacité du rééquilibrage du marché du travail (Greenwood, 1985).

Les coûts de migrations (monétaires et non monétaires) sont une variable de contrôle des politiques migratoires. Le coût fixe de départ est influencé par les contrôles aux frontières. Les coûts postérieurs, pendant le séjour du migrant, sont influencés par les politiques de permis de séjour, de lutte contre l'immigration clandestine, et le "climat général" du pays d'immigration. La littérature sur les migrations insiste assez largement sur les effets de réseaux qui peuvent réduire ces coûts psychiques en améliorant l'intégration. L'impact est assez complexe, car les coûts de migration ont tendance à varier négativement avec le nombre de compatriotes de même nationalité, jusqu'à un certain point, où apparaissent au contraire des effets de congestion: les coûts augmentent alors avec le nombre d'émigrés 
(Saint-Paul, 1997). Dans des comparaisons de données de niveau macro, où l'on ne peut pas distinguer la nationalité des immigrants et des étrangers précédemment immigrés, les retards dans le processus de migration permettent d'appréhender certains de ces aspects. En partie parce que l'information est coûteuse et qu'elle requiert du temps à être acquise, la migration répond avec retard aux variations (Greenwood, 1985). L'inertie dans la décision de migrer est lié à l'incertitude quant aux conditions alternatives du pays de destination. Si une destination est jugée préférable par un individu ou un groupe qui y émigre par la suite, un "conduit" pour le flux d'information vers le pays d'origine est établi. Ce conduit réduit le coût d'information concernant les alternatives et doit réduire l'incertitude à y émigrer (Stark et Bloom, 1985). Le regroupement familial explique aussi que des flux d'émigrants femmes et enfants suivent avec un certain retard des flux d'émigrants hommes ${ }^{8}$. Dans les études utilisant des données de niveau macro décrites par Stark et Bloom, les retards dans les flux ou taux d'émigration sont significatifs et de l'ordre d'un an. Depuis, toutes les autres études comparatives décrites ci-dessous incluent des retards de ce type.

La formulation d'anticipations adaptatives vis-à-vis de ces flux d'informations permet donc de dynamiser le modèle de l'équation $(5)^{9}$.

$$
\left(\frac{t M_{i, t}}{t / M_{i, t-1}}\right)=\left(\frac{t M_{i, t}^{e}}{t / M_{i, t-1}}\right)^{\left(1-\lambda_{i}\right)}
$$

Le processus d'ajustement de la migration est dichotomique (équation 6). Le taux d'immigration d'une année sur l'autre s'ajuste au niveau espéré avec une inertie $\lambda$. L'immigration ne s'ajuste pas d'une manière instantanée aux variations des déséquilibres sur le marché du travail du fait des coûts psychiques de migration et de l'incertitude sur l'anticipation des conditions du pays d'accueil. Cette incertitude et donc les coûts associés peuvent être atténués par les réseaux d'amis ou de compatriotes précédemment établis.

\section{Les équations réduites à estimer}

La combinaison des équations (5) et (6) et la linéarisation donnent les équations réduites qui vont être estimées:

$$
\log \left(\frac{M_{i, t}}{P O P_{i, t}}\right)=\alpha_{i} \cdot \log \left(\frac{w_{i, t}}{W_{*, t}}\right)+\beta_{i} \cdot \log \left(\frac{U_{i, t}}{U_{*, t}}\right)+\delta_{i} \cdot \log \left(\frac{M_{i, t}}{P O P_{i, t}}\right)_{-1}+\eta_{i}+\varepsilon_{i, t}
$$

avec $i=1, \ldots k$ pays européens,

$I M_{i}=$ flux d'immigration dans un pays $i$,

$P O P_{i}=$ population totale d'un pays i,

$W_{i}=$ salaire du pays i d'immigration en PPA,

$W^{*}=$ salaire moyen pondéré de I'UE en PPA,

8. Drettakis (1976) a montré que les femmes originaires des pays du sud de l'Europe (Italie, Grèce, Espagne, Turquie, Portugal) émigraient avec retard en comparaison des hommes émigrant en direction de l'Allemagne. Les retards étaient différents selon la nationalité.

9. Dès les années soixante-dix, on a obtenu de meilleurs résultats pour des anticipations adaptatives ou extrapolatives que pour des anticipations statiques (Walsh, 1974, pour les migrations irlandaises en direction de l'Angleterre). 
$U_{i}=$ taux de chômage du pays i d'immigration,

$U^{*}=$ taux de chômage moyen pondéré de l'UE,

$\eta_{i}=$ effet fixe du pays i,

et $\varepsilon_{i, t}$ est le terme aléatoire.

L'équation d'émigration est équivalente:

$$
\log \left(\frac{E M_{i, t}}{P O P_{i, t}}\right)=\alpha_{i} \cdot \log \left(\frac{w_{i, t}}{W_{*, t}}\right)+\beta_{i} \cdot \log \left(\frac{U_{i, t}}{U_{*, t}}\right)+\delta_{i} \cdot \log \left(\frac{E M_{i, t}}{P O P_{i, t}}\right)_{-1}+\eta_{i}+\varepsilon_{i, t}
$$

avec $E M_{i}=$ flux d'émigration du pays i.

Le premier type de facteurs caractérisant les déséquilibres entre les marchés du travail est donc limité aux différences entre les salaires des pays d'accueil et ceux des autres pays européens, d'une part, et celles qui existent entre les taux de chômage, d'autre part (équations 7 et 8). Dans le cas des immigrants non-communautaires, ce sont les salaires entre leurs pays d'accueil et la moyenne des autres pays de l'Union européenne qui sont pris en compte, et non pas les salaires de leurs pays d'origine. Ce n'est donc pas la réponse à la question assez traditionnelle des raisons du départ, mais plutôt celle des raisons du choix du pays de destination, à l'instar des autres études comparatives (Pissarides et McMaster, 1990; Eichengreen, 1993; Attanasio et Padoa Schioppa, 1991). La méthodologie est identique pour déterminer les taux de chômage relatifs.

Les autres facteurs de nature plus structurelle tels que les différences d'aménités (environnement du pays, conditions climatiques, dotations en infrastructures, protection sociale...) ou les coûts de migration et d'information (qui seront atténués par les effets de réseaux associés à la présence de compatriotes déjà installés dans les pays de destination des migrants), sont mesurés à travers la prise en compte d'effets fixes et de degrés d'inertie des taux de migration.

Les effets fixes peuvent traduire les caractéristiques spécifiques des pays comme par exemple les dotations en infrastructure attirant les immigrants dans une région. Les effets de réseaux peuvent être associés à la présence de fortes inerties temporelles dans les flux migratoires. Par exemple, dans un pays où le flux d'immigrants se reproduit dans la même proportion d'une année sur l'autre quelque soit l'évolution du marché du travail, les déterminants structurels et de réseaux dominent. On utilise, généralement, le stock de migrants déjà installés dans un pays pour caractériser les réseaux lorsque I'on travaille sur des flux migratoires par nationalité : ainsi, les émigrants chinois sont plus incités à venir en France si une nombreuse population chinoise est déjà présente. Mais dans notre analyse comparative sur des flux migratoires de non-Européens, on ne peut pas prendre un stock de migrants non-Européens (dont la variabilité en plus est faible): les Chinois ne viendront pas parce qu'il y a une forte communauté maghrébine en France, par exemple. II faudrait donc avoir des statistiques donnant la ventilation précise entre les nationalités des migrants et des étrangers dans le pays d'accueil. Dans le cas présent, le taux de migration retardé d'une 
année est la meilleure variable pour approximer les effets de réseaux. L'inertie des flux migratoires est d'ailleurs utilisée dans les études comparatives précédemment citées et détaillées infra.

Ce modèle aide à voir s'il existe une hiérarchie ou une concurrence entre les variables du marché du travail et les variables structurelles (aménités et réseaux), et une complémentarité à l'intérieur de ces variables structurelles entre les effets fixes (aménités) et l'inertie de l'ajustement (coûts d'information et psychiques de migration inversement corrélés aux effets de réseaux). Auparavant, une analyse des corrélations entre les flux d'immigration et les flux d'émigration donne un premier aperçu de l'importance des variables du marché du travail.

\section{Des corrélations positives entre les flux d'émigration et les flux d'immigration}

Pour effectuer le calcul des corrélations et des estimations économétriques, les données de migrations utilisées sont celles qui proviennent de la base New Cronos et les flux d'émigrants ne sont connus que pour les sept pays qui utilisent un registre de population (ANNEXE 1).

Si le salaire ou le chômage relatif expliquait essentiellement les évolutions des flux migratoires, la corrélation entre les flux d'immigration d'un pays et ses flux d'émigration serait négative. Une augmentation du salaire du pays d'accueil comparé à celui des autres pays provoquerait une baisse de l'émigration de ce pays car les résidents seraient moins incités à partir. Par contre, les flux d'immigrants évolueraient en sens inverse et seraient en croissance. La corrélation positive signifie, au contraire, que les deux flux évoluent dans le même sens et que les principaux déterminants des migrations ne sont pas liés aux déséquilibres sur les marchés du travail ${ }^{10}$.

Pour la majorité des pays européens, les flux d'immigration et d'émigration évoluent dans le même sens (TABLEAU 3). Sur les 33 calculs effectués, $60 \%$ des corrélations sont positives. Dans $20 \%$ des cas la corrélation est nulle et seulement $20 \%$ des corrélations sont négatives ${ }^{11}$. En Europe, les flux migratoires n'évoluent donc pas en sens inverse, mais plutôt de concert, en particulier les flux des communautaires (presque tous les coefficients sont positifs et élevés car compris entre 0,75 et 0,92). La Belgique fait exception puisqu'elle présente une corrélation nulle entre les deux flux des communautaires et des corrélations négatives pour les autres flux. La corrélation est aussi négative entre les flux de non-communautaires aux Pays-Bas. Les autres corrélations négatives (autour de - 0,5) concernent exclusivement les flux migratoires de pays périphériques (Espagne, Irlande, Portugal). Déjà, dans cette analyse sommaire semble naître une différence entre les comportements migratoires concernant les pays du centre et ceux de la périphérie de l'Union européenne.

10. Voir à ce sujet la controverse entre d'une part Althaus et Schachter (1989) et d'autre part Evans (1990), Greenwood et alii (1991). Rappelons aussi que l'introduction de I'hétérogénéité de la main-d'œuvre remet en cause ce lien négatif (Sjaastad, 1962).

11. Sept coefficients de corrélation sur trente-trois sont inférieurs à 0,25 (et inversement pour les coefficients positifs). Le manque de données de flux d'émigration pour une grande majorité des pays périphériques pourrait relativiser ce résultat. 
Tableau 3 - Coefficients de corrélation entre les flux d'immigrants et d'émigrants sur la période entre 1985 et 1999

\begin{tabular}{lcccc}
\hline & Total & Communautaires & $\begin{array}{c}\text { Non } \\
\text { Communautaires }\end{array}$ & Nationaux \\
\hline Belgique & $-0,49$ & 0,06 & $-0,35$ & $-0,55$ \\
Danemark & 0,84 & 0,87 & 0,45 & $0,65^{\star}$ \\
Allemagne & 0,43 & 0,74 & 0,62 & 0,61 \\
Espagne & & & & $-0,43^{* *}$ \\
Irlande & $-0,55^{* *}$ & & & $0,82^{* *}$ \\
Italie & & & & \\
Luxembourg & $0,93^{* * *}$ & & $-0,58$ & 0,08 \\
Pays-Bas & 0,31 & 0,42 & & \\
Portugal & $-0,55^{* * * *}$ & & 0,25 & 0,28 \\
Finlande & $-0,15$ & 0,92 & 0,71 & 0,92 \\
Suède & 0,08 & 0,84 & 0,09 & $-0,04$ \\
Royaume-Uni & 0,25 & 0,73 & & \\
\hline
\end{tabular}

*1985-1997, **1987-1997, ***1994-1999, ****1992-1999.

Note: Le coefficient de corrélation est utilisé pour déterminer la relation entre deux séries différentes:

Correl $(X, Y)=\frac{\sum(X-\bar{X})(Y-\bar{Y})}{\sqrt{\sum(X-\bar{X})^{2} \sum(Y-\bar{Y})^{2}}}$

où $\bar{X}$ et $\bar{Y}$ sont les moyennes échantillonnées.

Source: Eurostat (New Cronos), calculs des auteurs.

En première conclusion, pour la majorité des pays anglo-saxons de I'Union européenne, les variables de déséquilibre sur le marché du travail ne semblent pas être essentiels dans le choix de la destination des émigrants. Par contre, ces variables du marché du travail peuvent influencer sensiblement les choix en direction des pays périphériques.

\section{De fortes inerties dans les flux migratoires dans l'espace européen}

Au contraire des études comparatives précédentes (Pissarides et Mac Master, 1990; Attanasio et Padoa Schioppa, 1991; Eichengreen, 1993) qui ont estimé des taux de migrations nettes, nous distinguerons les taux d'immigration et ceux d'émigration afin de voir s'il existe des différences entre les comportements des immigrants et des émigrants et aussi en fonction de la nationalité d'origine. Dans le modèle d'Eichengreen (1993) ${ }^{12}$, l'immigration nette dépend positivement du salaire relatif et négativement du chômage relatif. La sensibilité des migrations aux variables du marché du travail est bien plus élevée aux États-Unis qu'au Royaume-Uni et en Italie où la migration nette est davantage expliquée par les effets d'inertie ou de réseaux. Près de $60 \%$ du solde migratoire se répète d'une année sur l'autre dans ces deux pays européens alors qu'aucune inertie n'apparaît dans le cas des États-Unis.

12. Cette étude s'inspire du modèle de Pissarides et Mac Master (1990) et Attanasio \& Padoa Schioppa (1991). 
En revanche, dans ce dernier pays, la sensibilité de la migration nette par rapport aux différences de salaires et de chômage est beaucoup plus forte qu'au Royaume-Uni ${ }^{13}$.

On retrouve cette plus grande sensibilité américaine aux variables du marché du travail dans I'étude de Mazier, Oudinet et Saglio (2002). II n'y a pas d'inertie d'un an sur l'autre dans les flux migratoires entre les quatre grandes régions américaines mais des effets fixes spécifiques aux régions attirent plus ou moins les migrants. Ce sont les perspectives d'emploi relatives et non pas le taux de chômage relatif qui sont fondamentales dans l'attraction des immigrants ${ }^{14}$. L'augmentation du revenu relatif de la région d'accueil tend à attirer des immigrants (élasticité de 0,9) mais n'a pas d'impact sur le comportement des émigrants. Enfin, il y une convergence entre les comportements migratoires puisque les élasticités liées aux variables du marché du travail sont identiques aux quatre régions.

Les équations sont estimées simultanément avec des variables indépendantes en apparence, dans le sens où aucune variable exogène dans une équation d'immigration d'un pays n'est endogène dans l'équation d'immigration d'un autre pays. Par contre, leurs perturbations sont corrélées (hétéroscédasticité des erreurs), ce qui implique que les variables expliquées ne sont plus indépendamment distribuées. La méthode SUR utilisée permet de pallier le problème de simultanéité et de tenir compte de la corrélation des perturbations. On teste ensuite des restrictions sur les coefficients afin de déterminer si les coefficients doivent être spécifiques aux pays ou communs à l'ensemble de l'Union européenne (voir l'ANNEXE 2 pour plus de détails).

Un premier résultat général montre que les migrants dans l'espace européen sont moins sensibles aux variables du marché du travail qu'aux déterminants structurels des flux migratoires tels que les coûts de migration et effets de réseaux associés (degré d'inertie du taux de migration) ou les différences d'aménités (effets fixes). Mais les comportements diffèrent selon les types de flux d'émigration ou d'immigration.

Pour les taux d'immigration, les deux variables explicatives caractérisant le déséquilibre sur le marché du travail ayant des élasticités significativement différentes de zéro ont un signe cohérent avec la théorie pour la plupart des pays. Ainsi une augmentation du salaire relatif du pays d'accueil provoque un accroissement du taux d'immigration alors qu'une augmentation du taux de chômage relatif du pays d'accueil (caractérisant une baisse des perspectives d'emploi dans ce pays d'accueil, ou une hausse des perspectives dans les pays d'origine) entraîne une baisse du taux d'immigration de ce pays d'accueil.

La ventilation par pays d'accueil confirme la grande diversité de comportements que l'on avait perçu supra même si globalement, les flux d'immigration dans les pays de l'Union européenne apparaissent moins sensibles aux variations du taux de chômage relatif qu'aux différences de salaires relatifs (TABLEAU 4) ${ }^{15}$. Les élasticités sont en effet d'un niveau assez

13. L'élasticité aux salaires relatifs est 25 fois plus forte aux États-Unis qu'au Royaume-Uni dans l'étude d'Eichengreen (1993), alors qu'elle n'était que cinq fois plus forte dans l'estimation de Pissarides et Mac Master (1990). L'élasticité au chômage relatif est aussi très élevée aux États-Unis (deux fois plus forte).

14. Les semi élasticités calculées s'avèrent assez élevées $(3,5$ et 3,8$)$.

15. Les résultats des tests de Wald indiquent qu'il faut relâcher les contraintes sur les coefficients et donc avoir des paramètres spécifiques par pays (tests disponibles auprès des auteurs). 
faible, ce qui signifie que l'impact de ces variables du marché du travail sur les flux d'immigration est limité. C'est en Finlande et au Royaume-Uni que l'immigration se révèle la plus sensible aux déséquilibres sur le marché du travail, car la sensibilité s'exprime conjointement sur les salaires et sur le taux de chômage. En Allemagne, au Danemark et en France, les salaires ont une plus grande influence, alors que les variations du taux de chômage sont dominantes en Espagne, au Portugal et à un degré moindre aux Pays-Bas.

En ce qui concerne, les variables structurelles, I'inertie la plus forte dans l'ajustement des migrations se trouve en Irlande, Finlande, Luxembourg, Allemagne et Belgique. Par contre, les effets fixes ont un impact négatif (hormis en Espagne) sur l'immigration au Portugal, en Grèce, en France, au Portugal et en Italie. II semble y avoir une complémentarité entre ces deux variables structurelles puisque les effets fixes négatifs les plus importants se retrouvent dans les pays qui n'ont pas de grande inertie.

Pour les flux d'émigration, seuls les pays ayant un registre population disposent de données disponibles dans la base New Cronos d'Eurostat. Ce sont les pays scandinaves et anglosaxons qui ont malheureusement des caractéristiques proches, hormis la Finlande située à la périphérie de l'Europe.

Les variables de déséquilibre du marché du travail ont encore moins d'influence sur les comportements des émigrants qu'elles n'en avaient sur ceux des immigrants. Ainsi le salaire relatif n'apparaît pas du tout comme une variable explicative significative: les émigrants ne quittent pas ces pays anglo-saxons pour des salaires plus élevés (TABLEAU 5). Le taux de chômage relatif ne joue que très légèrement son effet répulsif en Allemagne, Finlande et pas dans les autres pays. On voit là aussi une complémentarité entre l'inertie et les effets fixes ${ }^{16}$. C'est au Royaume-Uni que les aménités retiennent le plus les émigrants.

En définitive, les variables de déséquilibre du marché du travail ont encore moins d'influence dans la détermination des flux d'émigration que pour les flux d'immigration. Les facteurs structurels et les effets de réseaux semblent dominer le marché du travail dans le choix des émigrants. Pour les pays, où l'on peut comparer l'inertie de l'émigration avec celle de l'immigration, on s'aperçoit que cette dernière est plus forte. Les coûts de migration sont plus élevés à l'immigration dans les pays européens qu'à l'émigration.

Il faut tout de même rappeler que les flux globaux d'émigration concernent plusieurs composantes aux caractéristiques bien différentes. Si l'on se réfère à nos résultats précédents, la composante principale est celle des départs de nationaux d'un pays $A$ vers un pays $B$ que l'on va traiter dans la section suivante (et dans le tABLEAU 6). Les deux autres composantes de ces flux d'émigration, correspondant aux situations de migrations répétées (départs de non nationaux du même pays $A$ vers un pays B), seront analysées dans la seconde partie de l'article (et dans les tABLEAux 10 et 11).

16. C'est en Finlande que le degré d'inertie est le plus élevé (mais il est compensé par une absence d'effet fixe) et au Danemark que les effets fixes sont les plus importants (eux aussi compensés par une inertie non significative). 


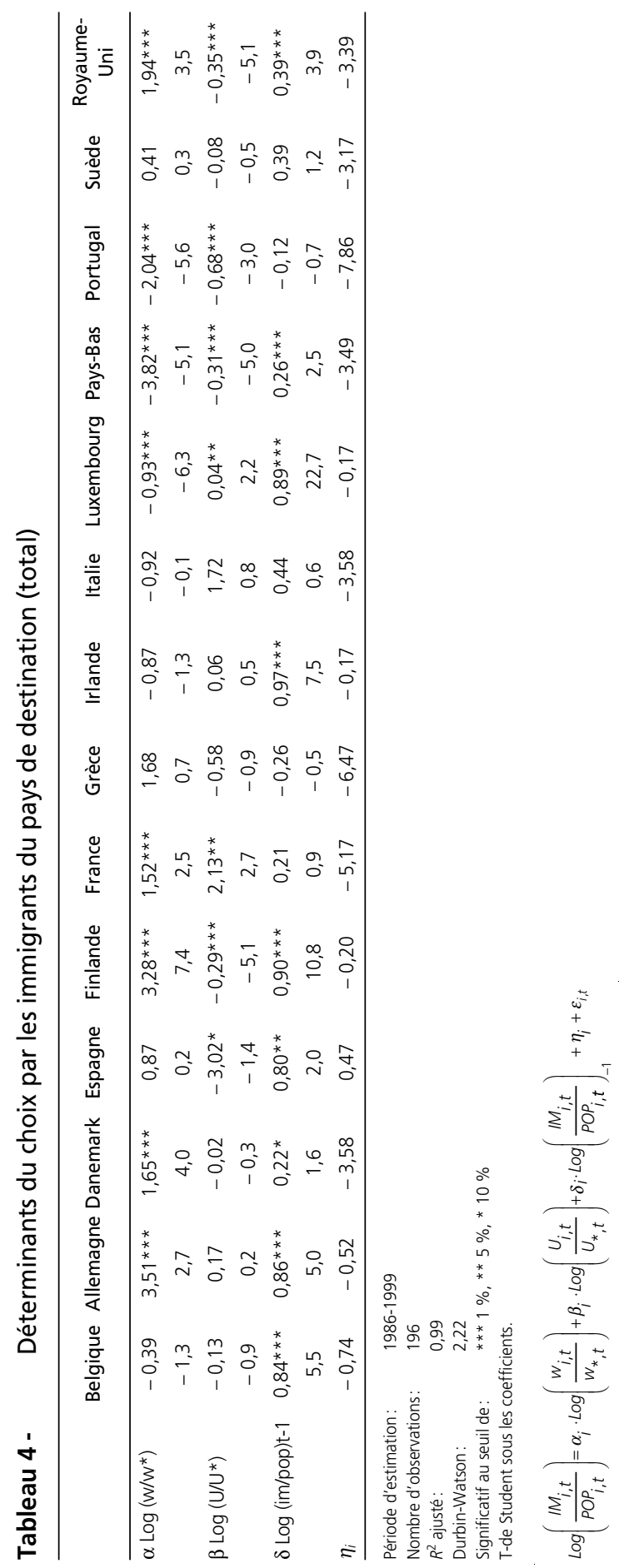


Tableau 5 - Déterminants du choix par les émigrants du pays de destination (total)

\begin{tabular}{lccccccc}
\hline & Belgique & Allemagne & Danemark & Finlande & Pays-Bas & Suède & $\begin{array}{c}\text { Royaume- } \\
\text { Uni }\end{array}$ \\
\hline$\beta \log \left(\mathrm{U} / \mathrm{U}^{*}\right)$ & $-0,26$ & 0,31 & $-0,05$ & $0,17^{* * *}$ & 0,03 & $0,12^{* * *}$ & 0,03 \\
& $-0,3$ & 1,2 & $-0,3$ & 5,3 & 0,6 & 2,5 & 0,4 \\
$\delta \log ($ em/pop)t-1 & $0,69^{* *}$ & $0,45^{* * *}$ & 0,24 & $0,99^{* * *}$ & $0,58^{* * *}$ & $0,62 * * *$ & $-0,06$ \\
& 2,4 & 2,9 & 1,5 & 8,7 & 2,7 & 4,2 & $-0,5$ \\
$\eta$ & $-1,7$ & $-2,6$ & $-3,8$ & 0 & $-2,4$ & -2 & $-5,9$ \\
\hline
\end{tabular}

Période d'estimation: 1986-1999

Nombre d'observations: 98

$R^{2}$ ajusté: $\quad 0,94$

Durbin-Watson: $\quad 2,01$

Significatif au seuil de: $\quad * * * 1 \%, * * 5 \%, * 10 \%$

T-de Student sous les coefficients

$\log \left(\frac{E M_{i, t}}{P O P_{i, t}}\right)=\beta_{i} \cdot \log \left(\frac{U_{i, t}}{U_{*}, t}\right)+\delta_{i} \cdot \log \left(\frac{E M_{i, t}}{P O P_{i, t}}\right)_{-1}+\eta_{i}+\varepsilon_{i, t}$

\section{Les retours des nationaux déterminés par des variables structurelles}

Comparés à l'ensemble de la population, les départs des nationaux (TABLEAU 6) sont beaucoup plus sensibles au taux de chômage relatif, et particulièrement en Belgique et en Allemagne, où l'élasticité est élevée. Les Belges et les Allemands qui partent de leurs pays semblent poussés par l'évolution du chômage relatif, ce qui n'est pas le cas pour les autres pays étudiés.

En effet, une augmentation d'un point du taux de chômage entraîne, toutes choses égales par ailleurs, dans ces deux pays, une augmentation équivalente du taux d'émigration des nationaux. Mais, dans le cas des nationaux également, le salaire relatif ne joue aucun rôle.

Tableau 6 - Déterminants du choix par les émigrants nationaux du pays de destination

\begin{tabular}{lccccccc}
\hline & Belgique & Allemagne & Danemark & Finlande & Pays-Bas & Suède & $\begin{array}{c}\text { Royaume- } \\
\text { Uni }\end{array}$ \\
\hline$\beta \log \left(\mathrm{U} / \mathrm{U}^{*}\right)$ & $1,2^{* * *}$ & $1,07^{* *}$ & 0 & $0,13^{* *}$ & 0,02 & $0,24^{* * *}$ & 0,17 \\
& 3,1 & 2,3 & 0 & 2,2 & 0,4 & 6,5 & 1,1 \\
$\delta \log (\mathrm{em} /$ pop $) t-1$ & $0,33^{* *}$ & 0,23 & $0,51^{* * *}$ & $1,01^{* * *}$ & $0,65^{* * *}$ & $0,67^{* * *}$ & 0,3 \\
& 2,1 & 1,3 & 4,3 & 6,8 & 4 & 10,3 & 1,3 \\
$\eta$ & $-4,3$ & $-4,9$ & $-2,6$ & 0,1 & $-2,1$ & $-1,9$ & $-4,3$ \\
\hline
\end{tabular}

Période d'estimation: 1986-1999

Nombre d'observations: 98

$R^{2}$ ajusté: $\quad 0,93$

Durbin-Watson: $\quad 1,73$

Significatif au seuil de: $\quad * * * 1 \%, * * 5 \%$, $10 \%$

T-de Student sous les coefficients.

$\log \left(\frac{E M_{i, t}}{P O P_{i, t}}\right)=\beta_{i} \cdot \log \left(\frac{U_{i, t}}{U_{*}, t}\right)+\delta_{i} \cdot \log \left(\frac{E M_{i, t}}{P O P_{i, t}}\right)_{-1}+\eta_{i}+\varepsilon_{i, t}$ 
Les effets fixes négatifs sont importants, particulièrement en Belgique, Allemagne et Royaume-Uni où les structures favorables de la société limitent l'incitation à émigrer.

Les retours des nationaux (TABLEAU 7) sont diversement sensibles aux variables du marché du travail. II semble y avoir une différence entre les pays du sud de l'Europe, pour qui les retours ne sont pas du tout sensibles aux salaires (ou bien de signe contraire) et aux variations de chômage, et les pays nordiques (Finlande, Suède, Danemark) le Royaume-Uni et la Belgique, pour qui les revenus sont significatifs. C'est en Suède que l'élasticité des retours au salaire est la plus élevée (2,82). Westerlund (1997) qui avait trouvé un résultat similaire, I'avait expliqué par l'existence d'un encadrement des salaires.

Dans les autres pays, les décisions de retour dans le pays d'origine sont bien souvent prises à l'avance, comme les retours en fin d'activité, et sont donc de nature structurelle, mais elles ne sont souvent pas faciles à prendre en compte comme en témoignent les degrés d'inertie élevés en Italie, en Irlande, ou bien les faibles aménités qui incitent peu aux retours en Grèce et au Portugal. Un autre effet mécanique peut aussi expliquer une continuité dans les flux de retour: les pays dont le taux d'émigration tend à baisser fortement voient leur flux de retour baisser quelques années plus tard, quelle que soit l'évolution du marché du travail. Ceci est particulièrement le cas des pays du Sud de l'Europe devenus récemment des pays d'immigration.

Une singularité est par contre à signaler: les retours des Espagnols et Finlandais sont sensibles aux variations du taux de chômage relatif. Dans ces pays, l'amélioration des conditions d'emploi, de vie et de niveau de salaire liée à leur rattrapage effectif par rapport aux pays du centre, explique une partie non négligeable du phénomène de retour des nationaux. En revanche, dans le cas du Portugal et de la Grèce, qui présentent encore des divergences structurelles par rapport aux autres pays de I'Union, les seuils des niveaux de salaire relatif demeurent trop faibles pour que leur variation conjoncturelle constitue un facteur incitatif suffisant aux retours des nationaux dans leur pays d'origine. Les retours s'expliquent davantage par des facteurs structurels, comme les aménités. 


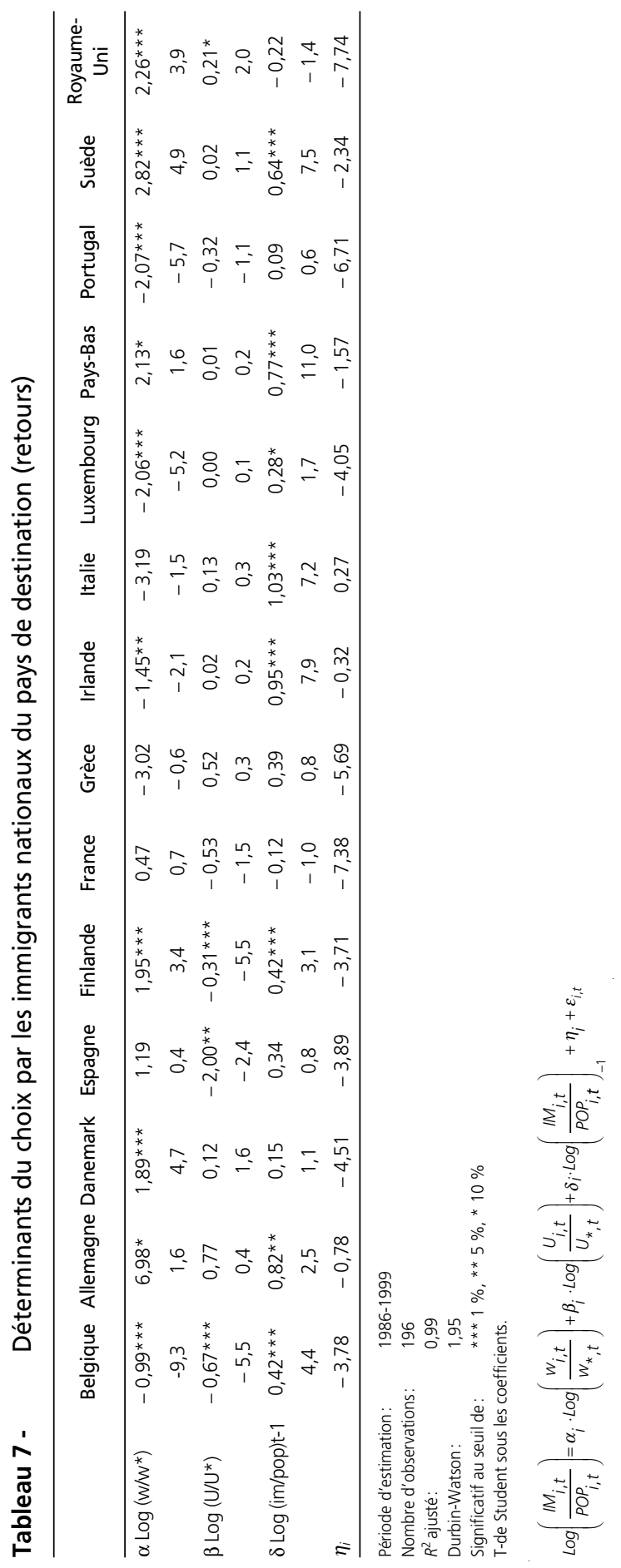




\section{LE RÔLE DU MARCHÉ DU TRAVAIL DANS LES CHOIX DE LOCALISATION DES MIGRANTS}

Cette section examine le rôle joué par les différences de salaires et de taux de chômage et par les effets de réseaux dans les choix de localisation des migrants selon qu'ils viennent des pays non membres de l'Union européenne ou des pays membres, et selon qu'ils sont ou non en situation de migration répétée.

\section{Les différences de comportement entre les communautaires et les non communautaires}

Une première analyse montre que les immigrants non-communautaires sont plus sensibles que les communautaires aux variables du marché du travail, en particulier aux différences de taux de chômage (TABLEAUx 8 et 9). Pour près de la moitié des pays européens, le taux de chômage est une variable significative pour les non-communataires (TABLEAU 9). Pour $40 \%$ des pays, le salaire est significatif, les migrants communautaires ne sont sensibles aux variables du marché du travail que dans quelques pays (Suède, Royaume-Uni en particulier, TABLEAU 8). Les décisions d'émigration par les non-communautaires se prennent davantage de manière spéculative, c'est-à-dire sans garantie préalable de trouver un emploi dans le pays d'accueil. Par contre, pour les migrants communautaires, plus souvent insérées dans des marchés internes aux firmes, leurs décisions d'émigrer sont prédéterminées en termes d'emploi (migrations contractées). Les migrants communautaires qualifiés conservent d'ailleurs souvent le même employeur (Bailly, Mouhoud et Oudinet, 2003). De fait, les coûts psychiques de migration sont moindres et l'inertie est en moyenne un peu plus faible pour les Européens. De même, l'impact négatif des effets fixes est moindre pour les communautaires ayant un accès facilité aux infrastructures publiques.

\section{Mobilité intra-firme pour les communautaires}

Les immigrants communautaires (TABLEAU 8) semblent plus sensibles à une hausse du salaire relatif lorsqu'ils vont dans les pays du nord (Suède, Danemark et Allemagne). Les raisons invoquées par Westerlund (1997), liées à l'encadrement des salaires expliquent la forte élasticité en Suède. Les perspectives d'emploi sont, par contre, prioritaires dans la décision de migrer au Royaume-Uni, en Belgique, ainsi qu'en Grèce. L'Espagne et le Portugal pourraient être dans le même cas, mais les coefficients ne sont pas suffisamment significatifs. Le cas du Royaume-Uni, connu pour attirer de nombreux jeunes Européens, est lui caractéristique, avec une élasticité trois fois plus importante pour les communautaires $(-1,2$ contre $-0,4$ pour les non-communautaires).

L'Espagne est par contre le seul pays qui a un effet fixe positif. Les aménités, dont le climat, incitent les communautaires à migrer en Espagne, et à un degré moindre en Irlande. Par contre, les coûts psychiques (liés à l'absence de réseaux) font que l'ajustement est le plus lent dans ces deux pays. Là encore, l'effet de complémentarité entre les deux types de variables structurelles apparaît. 


\section{Les pays d'accueil de la périphérie de l'Union européenne}

La plus forte sensibilité de l'immigration globale au taux de chômage en Espagne est essentiellement le fait des immigrants d'origine non-communautaire, dont l'élasticité y est la plus élevée (tableau 9). C'est aussi le cas en Finlande où de nombreux Russes et de ressortissants des pays de l'Europe de l'est sont les principaux migrants, et certainement en Grèce (albanais, bulgares et ex-yougoslaves) ${ }^{17}$. Ces trois pays qui sont des pays d'accueil et de transit pour les non-Européens, utilisent plus volontiers la migration comme variable d'ajustement.

Dans les pays caractérisés de pays cibles, les immigrants non communautaires sont aussi assez sensibles au marché du travail: plutôt au salaire, en Allemagne (essentiellement d'origine turque), en France (essentiellement d'origine maghrébine), ainsi qu'en Irlande, et plutôt au taux de chômage au Royaume-Uni, en Belgique et aux Pays-Bas. Au Portugal, on peut noter que les variables du marché du travail ne sont pas significatives pour l'immigration non-communautaire. L'organisation de l'immigration par les autorités et les entreprises portugaises (en provenance des PECO en particulier) en fonction des variations conjoncturelles explique cette faible dépendance aux variations du marché du travail. Les nouveaux flux d'immigrants (Ukrainiens et Européens de l'est) sont dépendants d'une politique déguisée de quotas (Mouhoud et Oudinet, 2003). Par contre, les faibles aménités pour eux expliquent un fort effet fixe négatif.

Les coûts psychiques sont plus élevés pour les non-communautaires dans la grande majorité des pays, en particulier en Espagne, Finlande. II n'y a qu'au Luxembourg, où la diaspora portugaise permet de les réduire, au point qu'ils soient moindres que pour un communautaire.

\section{Des migrants en situation répétée moins sensibles aux effets de réseaux}

La distinction opérée entre les émigrants étrangers (communautaires et non-communautaires) et les nationaux permet de comparer les comportements de ceux qui sont en migration répétée avec les autres (pour la typologie, voir ANNEXE 1). Par exemple, un émigrant communautaire ou non-communautaire qui quitte la Belgique, réalise au moins sa seconde migration. En effet, hormis ceux qui sont nés en Belgique (seconde génération), les étrangers qui repartent avaient déjà émigré une première fois pour arriver en Belgique précédemment. Les résultats de ces émigrations de communautaires (TABLEAU 10) et de non-communautaires (TABLEAU 11) peuvent ainsi être comparés avec ceux des émigrations de nationaux (TABLEAU 6).

Comme pour les émigrants nationaux, les variations de salaires ne déterminent pas les décisions des émigrants en situation répétée, qu'ils soient d'origine communautaire ou non. D'autres raisons de nature plus structurelle dictent leurs décisions puisque les effets fixes négatifs sont plus importants que ceux des nationaux.

17. Mais les coefficients, bien qu'élevés, ne sont pas suffisamment significatifs. 

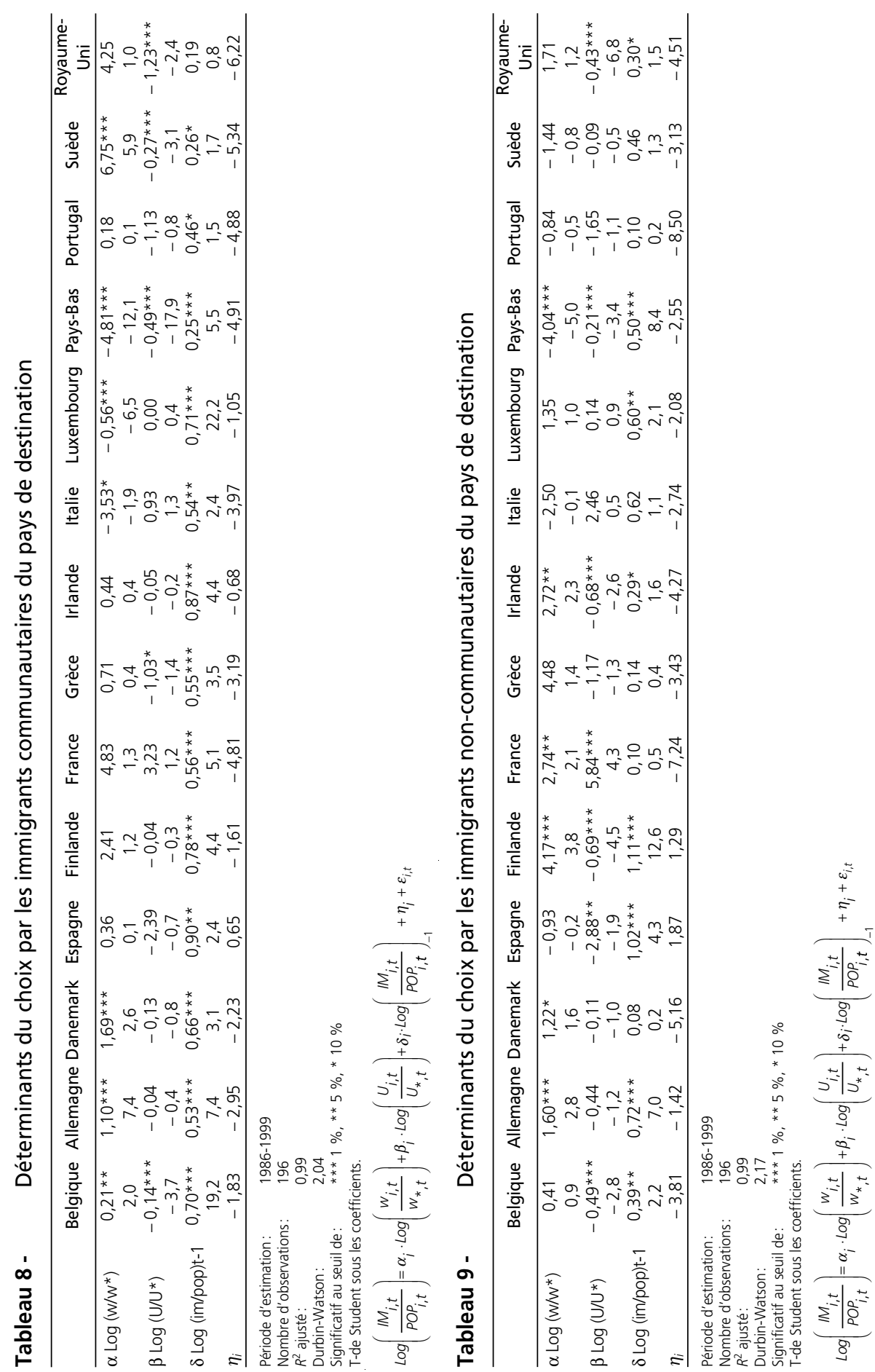
Tableau 10 - Déterminants par les émigrants communautaires du choix du pays de destination (migration répétée)

\begin{tabular}{|c|c|c|c|c|c|c|c|}
\hline & Belgique & Allemagne & Danemark & Finlande & Pays-Bas & Suède & $\begin{array}{c}\text { Royaume- } \\
\text { Uni }\end{array}$ \\
\hline \multirow[t]{2}{*}{$\overline{\beta \log \left(U / U^{*}\right)}$} & $2,05^{* * *}$ & $0,51 * *$ & $-0,54^{* * *}$ & $0,36^{* * *}$ & $-0,22^{* * *}$ & $0,17^{*}$ & $-1,39 * * *$ \\
\hline & 2,6 & 2,1 & $-2,6$ & 3,2 & $-2,6$ & 1,6 & $-4,9$ \\
\hline \multirow[t]{2}{*}{$\delta \log (e m / p o p) t-1$} & $-0,22$ & $0,49 * * *$ & $0,37 * *$ & $0,78^{* * *}$ & $-0,22$ & $0,54 * * *$ & $0,29 * *$ \\
\hline & $-0,8$ & 3,3 & 1,9 & 5,7 & $-1,1$ & 2,9 & 1,9 \\
\hline$\eta_{i}$ & $-7,7$ & $-3,2$ & $-4,8$ & -2 & $-9,2$ & $-3,4$ & $-5,7$ \\
\hline Période d'estimation: & 1986-199 & & & & & & \\
\hline Nombre d'observations: & s: 98 & & & & & & \\
\hline$R^{2}$ ajusté: & 0,95 & & & & & & \\
\hline Durbin-Watson: & 1,88 & & & & & & \\
\hline Significatif au seuil de: & $* * * 1 \%$ & ,** $5 \%, * 10 \%$ & & & & & \\
\hline T-de Student sous les co & coefficients. & & & & & & \\
\hline$\left(E M_{i, t}\right)=\beta$ & $\left(u_{i, t}\right)$ & & & & & & \\
\hline $\log \left(\frac{, i}{P O P_{i, t}}\right)=\beta_{i} \cdot \log$ & $g\left(\frac{1, l}{U_{*}, t}\right)+\delta$ & $i \cdot \log \left(\frac{1, l}{P O P_{i, t}}\right)$ & $+\eta_{i}+\varepsilon_{i, t}$ & & & & \\
\hline
\end{tabular}

Tableau 11 - Déterminants du choix par les émigrants non-communautaires du pays de destination (migration répétée)

\begin{tabular}{|c|c|c|c|c|c|c|c|}
\hline & Belgique & Allemagne & Danemark & Finlande & Pays-Bas & Suède & $\begin{array}{l}\text { Royaume- } \\
\text { Uni }\end{array}$ \\
\hline \multirow[t]{2}{*}{$\beta \log \left(U / U^{*}\right)$} & $-0,84$ & 0,04 & $-0,07$ & $0,36^{\star \star *}$ & $0,22 * *$ & $-0,12^{* *}$ & $0,81 * \star *$ \\
\hline & $-0,5$ & 0,1 & $-1,3$ & 3,1 & 2,0 & $-2,3$ & 4,0 \\
\hline \multirow[t]{2}{*}{$\delta \log ($ em/pop)t-1 } & $0,70^{*}$ & $0,46^{* * *}$ & $0,91 * * *$ & $-0,04$ & $0,43 * * *$ & $0,69 * * *$ & $-0,50 * *$ \\
\hline & 1,7 & 2,7 & 7,7 & $-0,2$ & 2,7 & 3,7 & $-2,5$ \\
\hline$\eta_{i}$ & $-2,1$ & $-2,8$ & $-0,6$ & $-8,8$ & $-4,0$ & $-2,2$ & $-10,1$ \\
\hline \\
\hline \multirow{2}{*}{\multicolumn{8}{|c|}{$\begin{array}{ll}\text { Nombre d'observations: } & 98 \\
R^{2} \text { ajusté: } & 0,96\end{array}$}} \\
\hline & & & & & & & \\
\hline & & & & & & & \\
\hline Significatif au seuil de: & $* * * 1 \%$ & $* * 5 \%, * 10 \%$ & & & & & \\
\hline T-de Student sous les coe & oefficients. & & & & & & \\
\hline$\left(E M_{i, t}\right)=\beta$ & $U_{i, t}$ & $E M_{i, t}$ & & & & & \\
\hline $\log \left(\overline{P O P_{i, t}}\right)=p_{i} \cdot \operatorname{LOg}$ & $p g\left(\overline{U_{*}, t}\right.$ & $9 \mid \overline{P O P_{i, t}}$ & $+\eta_{i}$ & & & & \\
\hline
\end{tabular}

En revanche, ils ont des comportements différents en fonction de leur nationalité. Les communautaires en situation de migration répétée sont un peu plus sensibles au chômage relatif que les non-communautaires. C'est en Belgique que ce phénomène est le plus marqué (la sensibilité est trois fois plus importante que celle des autres pays) et à un degré moindre en Allemagne. Alors qu'au Royaume-Uni et en Finlande, une augmentation du taux de chômage incite les non-communautaires à repartir. 
L'effet d'apprentissage lié à l'expérience de la migration semble avoir plus d'importance dans le cas des communautaires que dans celui des non-communautaires. Cela réduit leurs coûts psychiques de migration, et donc réduit l'inertie de la migration; c'est particulièrement le cas pour ceux qui sont passés au Royaume-Uni et en Suède. Les effets de réseaux sont moins déterminants pour les migrants communautaires en situation répétée car ces derniers subissent de manière moins intense les coûts psychiques de la migration dans l'espace socioculturel européen. Ils bénéficient d'un effet d'apprentissage lié à leurs premières expériences d'émigration qui diminue le coût de l'intégration dans le pays.

Au total, l'analyse des résultats des représentations fait apparaître deux effets:

- un effet de concurrence entre les variables du marché du travail et les variables structurelles (aménités et réseaux) d'une part;

- un effet de complémentarité à l'intérieur de ces variables structurelles entre les effets fixes (aménités) et l'inertie de l'ajustement (coûts d'information et psychiques de migration inversement corrélés aux effets de réseaux).

\section{CONCLUSION}

La migration intra-communautaire, même lorsque l'on intègre la mobilité des migrants nonEuropéens dans l'espace européen, ne peut pas jouer le rôle de facteur d'ajustement sur le marché du travail pour deux raisons essentielles, I'une d'ordre quantitative et l'autre d'ordre qualitative.

Tout d'abord, malgré la relance récente des flux d'immigration en Europe, persiste une faiblesse structurelle des flux intra-communautaires comparée à la mobilité entre les régions des États-Unis qui demeure environ dix fois plus forte. Ensuite, les facteurs déterminants de ces flux sont moins liés aux déséquilibres sur le marché du travail qu'aux effets de réseaux et de structures. On peut donc parler d'une prime aux effets de réseaux dans les déterminants des migrations au sein de l'espace européen au contraire de ce que l'on a pu observer dans le cas américain.

Néanmoins, on peut distinguer deux types de comportements sur les marchés du travail selon d'une part les catégories de migrants et d'autre part la position des pays d'accueil. Du point de vue des migrants, ceux qui viennent des pays non membres de l'Union européenne sont davantage influencés par les différences de salaires entre les pays dans leurs choix du pays d'accueil. Par contre, ceux qui viennent des pays membres sont moins sensibles aux salaires relatifs et à l'emploi puisqu'ils conservent le même emploi et le même salaire dans le cadre d'un marché interne du travail (firmes multinationales). Les migrants en situation répétée bénéficient d'un effet d'apprentissage de leurs premières expériences d'émigration qui diminue le coût de l'intégration dans le pays d'accueil.

La position du pays d'accueil intervient également dans la distinction des différentes logiques migratoires dans l'espace européen. Les pays périphériques de I'Union européenne, en situa- 
tion de divergence structurelle par rapport aux pays du centre, utilisent plus volontiers la migration comme variable d'ajustement ce qui explique le fait que les migrants sont davantage influencés par les différences de salaires et d'emplois en se rendant dans ces pays. À l'inverse les effets de réseaux et de structure jouent un rôle plus grand dans le cas de l'immigration dans les pays du centre de l'Union européenne.

E. M. M. \& J. O. ${ }^{18}$

\section{AnNeXe 1 \\ Les données sur les migrations la base New Cronos et les apports de l'enquête LFS}

Différents types de données sont utilisés dans cette étude: des séries directement issues des bases de données relatives aux migrations internationales New Cronos d'Eurostat, et des données d'enquêtes sur les forces de travail d'Eurostat (Labor Force Survey, LFS).

La collecte des données New Cronos est opérée par Eurostat ${ }^{19}$ à partir de sources nationales dont les critères d'enregistrement et les définitions des migrants sont hétérogènes. Aux différences de critères, viennent s'ajouter des définitions diverses de migrants en fonction de leur durée de résidence. Par exemple, certains pays (Belgique, Danemark, Finlande, Allemagne, Luxembourg, PaysBas et Suède) utilisent leurs registres de population ou leurs registres d'étrangers alors que d'autres utilisent leurs permis de résidence ou leurs permis de travail renouvelables (France, Grèce, Italie, Portugal, Autriche). Pour ces derniers, on comprend que les données d'émigration sont souvent absentes. Le Royaume-Uni, I'Irlande, l'Espagne utilisent encore d'autres sources (enquêtes). À ces différences de critères, viennent s'ajouter des définitions diverses de migrants en fonction de leur durée de résidence. Selon les recommandations des Nations-Unies, beaucoup de pays retiennent une durée de douze mois, mais certains ne s'y conforment pas. Pour la Belgique, I'Allemagne et le Luxembourg, trois mois suffisent, six mois pour les Pays-Bas. Les recensements permettent bien d'évaluer précisément et de façon exhaustive les effectifs d'étrangers mais leurs périodicités longues et différentes (de cinq à dix ans) empêchent de disposer de données continues.

Par contre, les enquêtes annuelles sur les forces de travail (Labor Force Survey - LFS) fournissent ces données chronologiques (Eurostat, 1998, 2002) pour la population de plus de quinze ans. Elles présentent aussi le grand avantage d'être homogènes entre les pays tant dans les définitions des variables que dans les critères.

L'enquête actuelle sur les forces de travail est réalisée par des entretiens. La base de sondage est fondée sur les registres de population pour la Belgique, le Danemark, I'Italie, le Luxembourg, la Suède et la Finlande, ou sur les recensements comme en Grèce, en Espagne, en France, en Irlande, en Autriche et au Portugal. En Allemagne, ces deux sources sont à la fois utilisées. Enfin,

18. Les auteurs remercient les rapporteurs pour leurs commentaires qui ont contribué à améliorer l'article.

19. Certaines données manquantes dans la base New Cronos sont complétées à l'aide de la base SOPEMI (OCDE) en particulier les données suivantes: flux d'immigration au Portugal des migrants européens et non-européens pour I'année 1996 et retour des nationaux (1996-1999); Irlande: européens et non-européens pour 1994-1999; France: européens pour 1999 et non-européens pour 1996-1999; Grèce: non-européens pour 1998. Les flux d'émigration à partir du Danemark des Européens et non-Européens pour l'année 1994. 
les Pays-Bas et le Royaume-Uni recourent aux adresses postales. La taille de l'échantillon est variable selon les pays. Le tABlEAU A1.1 indique les différentes tailles d'échantillon, les taux globaux de sondage par rapport à la population totale, et le taux de réponse aux sondages. Par ailleurs, le niveau de désagrégation concernant certaines caractéristiques des étrangers est parfois tellement fin que le faible nombre des observations doit être interprété avec prudence. Lorsque certaines réponses ne sont pas complètement fiables (car inférieures au premier seuil de fiabilité), une parenthèse l'indique.

Nous avons choisi d'utiliser essentiellement l'enquête sur les forces de travail réalisée par Eurostat (LFS) pour la première section. En revanche, on ne peut pas reconstituer des flux d'émigration à partir de ces données d'immigration: ainsi, les immigrants intra-communautaires lorsque leur nombre est faible, ne sont pas distingués par nationalité mais agrégés dans une catégorie dite "autres Européens" ce qui limite la possibilité de reconstruire des flux d'émigration à partir de la base LFS. En outre, les flux d'émigration des non-Européens ne sont pas, par construction, disponibles dans cette enquête. Cette dernière permet d'appréhender la population étrangère selon deux critères: celui de la nationalité au moment de l'enquête et celui du pays de naissance. On ne peut donc pas repérer la population immigrée sur la base de la définition démographique consistant à comptabiliser les personnes nées à l'étranger et de nationalité étrangère à la naissance. Le croisement de ces deux critères avec celui du pays de résidence l'année précédente permet d'appréhender les flux migratoires intra-communautaires et extra-communautaires.

Tableau A1.1 - Taille de l'échantillon de l'enquête LFS

\begin{tabular}{lccc}
\hline & Taille de l'échantillon & $\begin{array}{c}\text { Taux global } \\
\text { de sondage (en \%) }\end{array}$ & $\begin{array}{c}\text { Taux } \\
\text { de réponse (en \%) }\end{array}$ \\
\hline Belgique & 35000 ménages & 0,9 & 85 \\
Danemark & 15600 individus & 0,4 & 72 \\
Allemagne & 150000 ménages & 0,45 & 98 \\
Grèce & 30000 ménages & 0,87 & $90-92$ \\
Espagne & 65000 ménages & 0,5 & 91 \\
France & 75000 ménages & 0,33 & 90 \\
Irlande & 39000 ménages & 3,3 & 93 \\
Italie & 75512 ménages & 0,36 & 95 \\
Luxembourg & 8500 ménages & 5 & 78 \\
Pays-Bas & 60000 adresses & 1 & $55-60$ \\
Autriche & 31500 logements & 1 & 79 \\
Portugal & 20000 ménages & 0,68 & 91 \\
Finlande & 19717 individus & 0,4 & 87 \\
Suède & 17000 individus & 0,3 & 87 \\
Royaume-Uni & 68250 ménages & 0,4 & 78 \\
\hline
\end{tabular}

Source: Eurostat (1998).

L'enquête sur les forces de travail permet d'appréhender la population immigrée selon le critère de la nationalité au moment de l'enquête et celui de la région de résidence l'année précédente (TABLEAU A1.2). Nous distinguons deux types de critère d'utilisation de l'enquête LFS:

- population mesurée selon le critère de nationalité;

- population mesurée selon le critère du pays de naissance. 
Selon le critère de la nationalité, trois catégories peuvent être définies:

- ceux qui possèdent la nationalité du pays d'accueil (nationaux);

- ceux qui ne possèdent pas la nationalité du pays d'accueil et qui ont la nationalité d'un pays membre de l'Union européenne (communautaires);

- ceux qui ne possèdent pas la nationalité du pays d'accueil et qui n'ont pas la nationalité d'un pays membre de l'Union européenne (non communautaires).

Ces deux dernières catégories permettent d'appréhender la population totale d'étrangers. Le critère de la région de résidence l'année précédente permet d'appréhender si la migration est intracommunautaire (la personne résidait dans un pays de I'Union européenne l'année précédente) ou extra-communautaire (la personne résidait dans un pays autre que l'Union européenne l'année précédente).

Tableau A1.2 - Typologie des catégories de migrants en Europe, à partir des données d'enquête LFS

En provenance d'un autre pays de l'Union européenne

(1)

Nationalité Union européenne

Européens en mobilité intracommunautaire
(3)

(5)

Nationalité non-Union européenne

Non-Européens en mobilité intracommunautaire (migration répétée)

Total Européens en mobilité intra-communautaire $(1+3)$

En provenance d'un pays extra-Union européenne

(2)

Nationalité Union européenne

Européens en mobilité extra-communautaire (migration répétée)
Nationaux

Retours intra-communautaires

Total Européens en mobilité extra-communautaire $(2+4)$

Note: Le cadre 1 du tableau indique que le migrant a la nationalité d'un des pays de l'Union européenne et qu'il résidait I'année précédente dans un pays de l'Union.

Le cadre 2 indique que le migrant a la nationalité d'un des pays de l'Union européenne mais qu'il résidait l'année précédente dans un pays hors de l'Union. 


\section{AnNeXe 2 \\ Méthodologie pour estimer les déterminants des migrations}

Les données macroéconomiques (salaire, prix à la consommation, PPA, taux de chômage, nombre de salariés, population) sont issues directement ou construites à partir de la base de données Economic Outlook de l'OCDE.

Les séries de flux d'immigration utilisées pour ces estimations sont issues de New Cronos et ont été reconstituées sur la période 1985-1999 pour 14 pays de I'Union européenne ${ }^{20}$. En ce qui concerne les séries de flux d'émigration, le panel est bien moindre compte tenu de l'absence de données dans les pays n'ayant pas de registre de population ou d'enquêtes. Seuls les flux d'émigration de sept pays scandinaves et anglo-saxons sont disponibles qui ont malheureusement des caractéristiques proches concernant les flux migratoires ${ }^{21}$.

La distinction des flux de migrants en fonction de leur nationalité d'origine (communautaires, non communautaires et nationaux) permet de repérer des comportements spécifiques liés à la nationalité. Une analyse des corrélations entre les deux flux migratoires est réalisée en premier. La confirmation d'une corrélation majoritairement positive nous conduit ensuite à estimer séparément les flux d'émigration et ceux d'immigration et non pas à les agréger dans une variable commune comme la migration nette (IM - EM).

Pour les estimations, les variables expliquées sont les taux d'immigration, soit le rapport du flux d'immigration sur la population totale (IM/POP), et les taux d'émigration, rapport du flux d'émigration sur la population totale (EMIPOP). Plusieurs variables représentatives des déséquilibres sur le marché du travail ont été construites et testées. Le salaire relatif en parité de pouvoir d'achat ${ }^{22}$ est le rapport du salaire en PPA du pays d'accueil sur le salaire en PPA des concurrents. Ici le salaire en PPA des concurrents est celui de la zone euro, calculé en pondérant chaque salaire en PPA par le pourcentage de salariés. Les salaires relatifs sont ceux du secteur privé (Compensation per Employee, Private Sector).

Rappelons que pour les non communautaires, l'élasticité du salaire relatif compare le salaire du pays d'accueil au salaire moyen de l'Union européenne, et non pas au salaire de leur pays d'origine. On se situe par là dans une logique d'ajustement où les immigrants non communautaires vont dans un des quinze pays européens. Les taux de croissance de l'emploi relatif et les taux de chômage relatif sont calculés par rapport aux taux de l'Union européenne. Ces variables caractérisent l'effet attractif ou répulsif (push and pull) du marché du travail.

Les autres variables explicatives des migrations non liées aux déséquilibres sur le marché du travail, comme les aménités (prise en compte de l'environnement du pays comme les conditions climatiques ou les dotations en infrastructures) ou comme les flux répétés de migrants d'une nationalité (rejoignant d'autres compatriotes déjà installés) seront prises en compte d'une manière globale soit à travers l'introduction d'effets fixes par pays, soit à travers des degrés d'inertie spécifiques

20. L'Autriche est absente de ce panel car les données ne sont disponibles qu'à partir de 1995.

21. Hormis la Finlande qui est aussi un pays à la périphérie de I'Union européenne.

22. La parité de pouvoir d'achat (PPA) est celle calculée par l'OCDE. 
des taux de migration. Ainsi des dotations en infrastructures peuvent attirer des migrants d'une manière structurelle dans une région. Une très forte inertie des flux migratoires démontre que ces migrations se renouvellent assez régulièrement quelle que soit l'évolution du marché du travail.

$$
\log \left(\frac{M_{i, t}}{P O P_{i, t}}\right)=\alpha_{i} \cdot \log \left(\frac{W_{i, t}}{W_{*, t}}\right)+\beta_{i} \cdot \log \left(\frac{U_{i, t}}{U_{*, t}}\right)+\delta_{i} \cdot \log \left(\frac{M_{i, t}}{P O P_{i, t}}\right)_{-1}+\eta_{i}+\varepsilon_{i, t}
$$

avec $i=1, \ldots k$ pays européens,

$M_{i}=$ flux d'immigration dans un pays i,

$P O P_{i}=$ population totale d'un pays i,

$W_{i}=$ salaire du pays $i d^{\prime}$ immigration en PPA,

$W^{*}=$ salaire moyen pondéré de I'UE en PPA,

$U_{i}=$ taux de chômage du pays i d'immigration,

$U^{*}=$ taux de chômage moyen pondéré de l'UE,

$\eta_{i}=$ effet fixe du pays i, et $\varepsilon_{i, t}$ est le terme aléatoire.

L'équation d'émigration est équivalente:

$$
\log \left(\frac{E M_{i, t}}{P O P_{i, t}}\right)=\alpha_{i} \cdot \log \left(\frac{W_{i, t}}{W_{*, t}}\right)+\beta_{i} \cdot \log \left(\frac{U_{i, t}}{U_{*, t}}\right)+\delta_{i} \cdot \log \left(\frac{E M_{i, t}}{P O P_{i, t}}\right)_{-1}+\eta_{i}+\varepsilon_{i, t}
$$

avec $E M_{i}=$ flux d'émigration du pays i.

Lorsque les équations forment un système à équations simultanées, la variable explicative n'est pas indépendante des perturbations et l'application des MCO à chacune des équations structurelles prise séparément donne des estimateurs biaisés et non-convergents et fournit des t-stat biaisées vers le bas.

Pour remédier au problème de simultanéité plusieurs méthodes dont les DMC (double moindres carrés), mais pour notre cas, on utilise la méthode SUR (seemingly unrelated regressions), qui s'avère plus pertinente que les DMC. Cette méthode s'applique à un cas particulier des systèmes à équations simultanées, car il s'agit de modèles où les équations sont indépendantes en apparence, mais elles sont en réalité liées par leurs perturbations. Elles sont indépendantes dans le sens où aucune variable exogène dans une équation n'est endogène dans une ou plusieurs autres, ce qui est le cas pour nos différentes équations d'immigration par pays. Par contre, leurs perturbations sont corrélées (hétéroscédasticité des erreurs), ce qui implique que les variables à gauche du signe égal ne sont plus indépendamment distribuées. La méthode permet à la fois de pallier au problème de simultanéité mais aussi de tenir compte de l'arbitrage en permettant aux perturbations d'être corrélées. On admet l'homoscédasticité des erreurs dans les séries temporelles lorsque les ordres de grandeur des variables sont voisins. Mais dans l'étude des données de panel, les variables peuvent avoir des valeurs très différentes. II y a alors hétéroscédasticité. La première possibilité pour réduire les biais des estimations est de normer les variables (en divisant par la même variable, on réduit les différences de grandeur, la variance résiduelle n'est plus dépendante de $x$ ): c'est ce que nous faisons en prenant les taux d'immigration plutôt que l'immigration. La taille de l'échantillon ne permet pas de faire un test significatif de l'autocorrélation et de I'hétéroscédasticité. Mais l'hypothèse d'hétéroscédasticité est plausible compte tenu des différence de taille entre les variances des résidus puisque nos pays européens sont grandement différents. 
Ensuite, il faut faire une hypothèse sur le poids relatif de chacune des observations car elles n'ont manifestement pas le même poids. Les petits pays européens ne doivent manifestement pas compter autant que les grands pays européens, et le constat est identique pour les régions américaines même si les différences sont moins criantes. Pour attribuer une pondération, la matrice de covariance est estimée dans une première régression et est appliquée dans des moindres carrés généralisés dans un second temps ${ }^{23}$. Cette méthode permet aussi de fixer des contraintes inter-équations sur les coefficients en fonction des résultats des tests emboîtés. Ainsi, on peut mettre en valeur des comportements spécifiques aux régions (le comportement des migrants est différente en fonction du pays d'accueil ou d'origine), alors que dans d'autres cas, le comportement des migrants est identique quel que soit le pays de départ ou d'arrivée. Dans un premier temps des contraintes inter-équations ont été fixées sur les coefficients des variables explicatives des déséquilibres sur le marché du travail afin d'avoir pour repère un coefficient global pour I'Union européenne ${ }^{24}$. La contrainte sur le coefficient unique est ensuite relâchée afin d'estimer des paramètres spécifiques aux pays. Seules ces équations sont commentées dans cet article, car en fonction des tests de Wald, il convient de relâcher la contrainte de coefficient unique.

\section{RÉFÉRENCES}

Althaus, P.-G., Schachter, J., 1989. An equilibrium model of gross migration, Journal of Regional Science 29 (2), mai, 143-159.

Attanasio, O.-P., Padoa-Schioppa, F., 1991. Regional inequalities, migration and mismatch in Italy, 1960-1986, dans Padoa-Schioppa, F. (Eds) Mismatch and Labor Mobility, Cambridge University Press, 237-321.

Bailly, F., Mouhoud, E., M., Oudinet, J., 2003. Union européenne: les nouvelles dynamiques migratoires: relance et complexification, Chronique internationale de I'IRES 84, numéro spécial, "Mouvements et politiques migratoires, les enjeux sociaux", septembre.

Bayoumi, T., Prasad, E., 1995. Currency union, economic fluctuations and adjustment: Some empirical evidence, Centre for Economic Policy Research, Discussion Paper Series 1172.

Blanchard, O.-J., Katz, L.-F., 1992. Regional evolution, Brookings Papers on Economic Activity 1, 1-61.

Blanchard O.-J., Muet, P.-A., 1993. Competitiveness through disinflation: An assesment of the French macroeconomic strategy, Economic Policy: A European Forum 8 (16), avril, 11-56.

Cadiou, L., Guichard, S., Maurel, M., 1999. La diversité des marchés du travail en Europe: quelles conséquences pour l'union monétaire?, CEPII, Document de travail 1999-11, juin.

Detang-Dessendre, C., Jayet, H., 2000. Migrations, équilibre et déséquilibre spatial, dans Baumont, Combes, Derycke, Jayet (Eds) Economie géographique, Economica, 15-31.

Drettakis, E.-G., 1976. Distributed lags models for the quarterly migration flows of West Germany, Journal of the Royal Statistical Society 139, Series A, Part 3, 365-373.

23. Une autre méthode consiste à réaliser une première estimation avec un poids égal et appliquer les résultats avec des moindres carrés pondérés dans un second temps (cross section weights).

24. Les résultats avec les coefficients contraints et donc communs à tous les pays sont disponibles dans Mouhoud et Oudinet (2003). 
Dumont, J.-C., Garson, J.-P., 2004. Immigration dans la zone OCDE: un panorama à multiples facettes, Revue française des affaires sociales 2, "Circulations migratoires", avril-juin.

Dumont, J.-C., Le Maitre, G., 2005. Beyond the headlines. New evidence on the brain drain, Revue économique 56 (6), 1275-1299, novembre.

Eichengreen, B., 1993. Labor markets and European monetary unification, dans Masson, P.-R., Taylor, M.-P. (Eds.) Policy Issues in the Operation of Currency Unions, Cambridge University Press, 130-162.

Eurostat, 1998. Enquête communautaire sur les forces de travail. Méthodes et définitions, Office statistique des Communautés européennes.

Eurostat, 2002. Statistiques sociales européennes. Résultats de l'enquête sur les forces de travail 2002, Office statistique des Communautés européennes.

Evans, A.-W., 1990. The assumption of equilibrium in the analysis of migration and interregional differences: A Review of some recent research, Journal of Regional Science 30 (4), 515-531, novembre.

Graves, P.-E., 1979. A Life cycle empirical analysis of migration and climate by race, Journal of Urban Economics 6 (2), 135-147, avril.

Graves, P.-E., 1980. Migration and climate, Journal of Regional Science 20 (2), 227-237, mai.

Greenwood, M.-J., 1975. Research on internal migration in the United States: A survey, Journal of Economic Litterature 13 (2), 397-433, juin.

Greenwood, M.-J., 1985. Human migration, theory, models and empirical studies, Journal of Regional Science 25 (4), 521-544, novembre.

Greenwood, M.-J, 1991. Migration, regional equilibrium, and the estimation of compensating differentials, American Economic Review 81 (5), 1382-1390, décembre.

Harris, J., Todaro, M., 1970. Migration, unemployment \& development: A two-sector analysis, American Economic Review 60 (1), 26-42, mars.

Jayet, H., 1996. L'analyse économique des migrations: une synthèse critique, Revue économique 3, 193-226.

Math, A., Rodier, C., 2003. Union européenne: communautarisation des politiques migratoires. Entre fermeture des frontières et besoins de main-d'œuvre, Chronique internationale de I'IRES 84, numéro spécial, "Mouvements et politiques migratoires, les enjeux sociaux", septembre.

Mazier, J, Oudinet, J, Saglio, S., 2002. La flexibilité des prix relatifs et la mobilité du travail en Union monétaire: une comparaison Europe-États-Unis, Revue de l'OFCE, Observatoire Français des Conjonctures Economiques, Paris, octobre.

Mouhoud, E.M., Oudinet, J. (sous la direction de), 2003. Les dynamiques migratoires dans I'union européenne: ajustements sur les marchés du travail et comparaison Europe-États-Unis, convention CEPN-MIRE, ministère de I'Emploi et des Affaires sociales, rapport final, 2 tomes.

Mundell, R., 1957. International trade and factor mobility, American Economic Review 47.

Pissarides, C-A., McMaster, I., 1990. Regional migration, wages and unemployment: Empirical evidence and implications for policy, Oxford Economic Papers 42 (4), 812-831, octobre.

Rendall, M.-S., Tomassini, C., Elliot, D.-J., 2003. Estimation of annual international migration from the Labour Force Surveys of the United Kingdom and the continental European Union, Statistical Journal 20 (3-4), 219-234. 
Saint-Paul, G., 1997. Economic integration, factor mobility and wage convergence, Centre for Economic Policy Research, Discussion Paper Series 1597.

Sjaastad, L., 1962. The costs and returns of human migration, Journal of Political Economy 70, 80-93.

Stark, O., Bloom, D.-E., 1985. The new economics of labor migration, American Economic Review 75 (2), 173-178, mai.

Walsh, B.-M., 1974. Expectations, information, and human migration: Specifying an econometric model to Britain, Journal of Regional Science 14 (1), 107-120, avril.

Westerlund, O., 1997. Employment, opportunities wages and interregional migration in Sweden 1970-1990, Journal of Regional Science 37 (1), 55-73, février. 
